\title{
La TV por IP en la Universidad: Un reto de divul- gación científica televisiva para jóvenes chilenos ${ }^{1}$
}

\author{
Daniel TorRALES-AGUIRRE \\ Universidad Católica del Norte (Chile) \\ dtorrale@ucn.cl \\ J. Ignacio AgUADED-GómEZ \\ Universidad de Huelva \\ ignacio@aguaded.es \\ Daniel PonCE-GuARDIOLA \\ Universidad de Huelva \\ ponceguardiola@gmail.com
}

Recibido: 20 de abril de 2013

Aceptado: 23 de septiembre de 2013

\section{Resumen}

Diseñar nuevos modelos de televisión en el contexto de la sociedad digital se convierte en una necesidad desde el ámbito de la investigación y la academia. El presente trabajo analiza los intereses y necesidades de información expresados por jóvenes espectadores chilenos, con el objeto de diseñar una IPTV universitaria, modalidad de TV por Internet, que incorpora, mediante las múltiples interacciones que genera Internet los contenidos audiovisuales preferidos por dicho público meta, con énfasis en lo cultural y educativo. Los jóvenes chilenos están de acuerdo en que el nuevo medio prevalecerá sobre los demás y contribuirá a la adquisición de nuevos saberes, procedimientos y utilidades para ser utilizados en la vida cotidiana, así como al incremento de la cultura científica y su participación ciudadana activa.

Palabras clave: IP-TV, WebTV, jóvenes, hábitos televisivos, innovación tecnológica.

\section{IP-TV in the University: A challenge popular science television for young Chileans}

\begin{abstract}
This paper analyzes the information needs and interests of young viewers in order to design an IP-TV University, Internet TV mode, to incorporate the advantages of the Internet and journalistic tools innovative audiovisual content preferred by the target audience, with emphasis on culture and education, especially produced for the web. The study used quantitative methodology, a survey, conducted on stratified probability sample (college students, young professionals, without higher education). Young people agree that the new medium will prevail and that it can contribute to the acquisition of knowledge, procedures and devices for use in their daily lives and that increase scientific literacy and active citizenship.
\end{abstract}

Keywords: IP-TV, WebTV, young, viewing habits, technological innovation.

1 Este trabajo contextualiza resultados en el marco del proyecto chileno de investigación (VRIDT-UCN 2010-12) «Creación e Implementación de una IPTV Universitaria. Incorporación de 5 Escuelas de Periodismo a la televisión digital vía Internet», y el proyecto de investigación andaluz «La competencia audiovisual de la ciudadanía andaluza. Estrategias de alfabetización mediática en la sociedad del ocio digital» (SEJ-5823). El grupo chileno de trabajo estuvo dirigido por el investigador Daniel Torrales, así como por Marcos Chait y Mauricio Matus (co-investigadores). Colaboraron los alumnos Pablo Mamani, Cristian Meneses, Jesús Montecinos, Rodrigo Ramos y Alejandro Rodríguez, de la Escuela de Periodismo UCN en la aplicación de encuestas. 


\section{Referencia normalizada}

TORRALES-AGUIRRE, Daniel; AGUADED-GÓMEZ, J. Ignacio; y PONCE-GUARDIOLA, Daniel (2014): "La TV por IP en la Universidad: Un reto de divulgación científica televisiva para jóvenes chilenos". Estudios sobre el Mensaje Periodístico. Vol. 20, Núm. 1 (enero-junio), págs.: 281-294. Madrid, Servicio de Publicaciones de la Universidad Complutense.

Sumario: 1. Introducción. 2. Estado del arte. 3. La IPTV y sus características distintivas. 4. Jóvenes y consumo de nuevos medios. 5. Material y métodos. 6. Análisis y resultados. 7. Discusión y conclusiones. 8. Referencias bibliográficas.

\section{Introducción}

Integrar Internet en la educación y con ello la TV, gracias a la convergencia digital, entendida como la aproximación de dispositivos de consumo, tales como el teléfono, la televisión y el ordenador, para la difusión de contenidos on-line (Corredoira, 1998:16) resulta una propuesta coherente con la sociedad de la información en la que vivimos y que está permitiendo pasar de «una televisión de penuria y de masa a una televisión de abundancia y de nicho» (Roux, 2007). En los últimos años, las universidades «han optado por incorporar el vídeo a los procesos de formación mediante el desarrollo de portales corporativos de televisión en Internet; con ello pretenden dar un impulso a la investigación y a la docencia mediante la integración y uso de medios audiovisuales. Muchas universidades españolas y latinoamericanas cuentan con servicios de distribución de vídeo por internet con la creación de portales web de TV universitaria» en unos caso y de IPTV en otros (Aguaded \& Ponce-Guardiola, 2012).

El uso que los jóvenes realizan de las tecnologías de información y comunicación (TIC) ha significado un aumento del consumo cultural, especialmente en cine y en música, según indica el estudio «Cultura y Juventud» (La Vanguardia, 2010). En Francia los jóvenes, especialmente entre 15 y 24 años, gustan de la actualidad, pero no se informan por la prensa tradicional y recurren a Internet para ver diarios («Le Monde», «Libération», «Aujourd'hui en France», «Le Figaro»), y la televisión, que es una importante fuente de información. Sin embargo, estiman que los noticieros televisivos no han evolucionado. Los consideran una pérdida de tiempo, con horarios incompatibles y aburridos. Además si 30\% de los jóvenes prefieren Internet para informarse - algo menos que la televisión (33\%) y más lejos de otros medios, como la prensa escrita $(17 \%)$ y radio $(10 \%)$ - solamente $12 \%$ tienen plena confianza en la Red; poseen certidumbre mayor (64\%) con la prensa escrita y la TV, $14 \%$. Por ende, deben hacerse cambios en las TIC, entre ellos el tratamiento de la información, para lograr más credibilidad por parte de los jóvenes.

En esta línea, la formación basada en competencias, incorporada en el modelo educativo europeo y también latinoamericano, es una propuesta viable de educación que parte del aprendizaje significativo y se orienta a la educación humana integral, como condición esencial de todo proyecto pedagógico. Contempla saber (conocimientos), saber hacer (habilidades) y saber ser (actitudes y valores). Dicha perspectiva (Tobón, 2004), integra la teoría con la práctica en las diversas actividades; promueve la continuidad entre todos los niveles educativos; fomenta la construcción del aprendizaje autónomo; orienta la formación y el afianzamiento del proyecto ético de vida y fun- 
damenta la organización curricular con base en planes y problemas, trascendiendo de esta manera el currículo estructurado en asignaturas compartimentadas.

La televisión por Internet, en sus modalidades de WebTV e IPTV, constituye una alternativa a la televisión digital terrestre (TDT) que en el caso de Latinoamérica, corresponde a la norma japonesa ISDB-T (Integrated Services Digital Broadcasting-Terrestrial). Ello especialmente para las Universidades que, con menos recursos económicos a invertir, en comparación con la TDT, pueden crear e implementar esos nuevos medios de comunicación, para emplearlos en la formación por competencias de los estudiantes de Periodismo en la gestión y realización de proyectos audiovisuales en la era digital (Torrales \& Matus, 2009: web). Desde la óptica tecnológica, pasar de una WebTV a una IPTV constituye una evolución significativa, ya que la segunda, a diferencia de la primera (de transmisión emisor-receptor), permite la comunicación peer-to-peer (par a par), entre usuarios activos, en este caso universidades y productoras independientes, posibilitando que las casas de estudios superiores sean tanto emisores como receptores, de una parrilla programática común. Ello faculta formar una comunidad virtual de aprendizaje para estudiantes, docentes, y realizadores audiovisuales externos, o bien una comunidad especializada, es decir «formas de sociabilidad construidas en torno a intereses específicos» (Castells, 2001). Además, al incluir las pequeñas empresas, se logra que la producción local y regional sea conocida por la comunidad. En ese contexto, Internet puede ser una alternativa para la difusión de esta producción, «permitiendo una democratización de la información que difícilmente será vista en los aparatos de televisión (Fagundes, 2006). Junto a ello, y también significativo, los abonados a la IPTV pueden, cumplidas las normas de calidad del medio, publicar sus propios materiales audiovisuales, siendo así no solo receptores, sino también emisores.

Las posibilidades tecnológicas y didácticas de la IPTV motivaron la puesta en marcha de un estudio que tuvo como objetivo detectar las potencialidades y expectativas de crear e implementar una IPTV universitaria, modalidad de televisión digital por Internet, que aglutina dimensiones periodísticas, tecnológicas y económicas, sustentable en el tiempo, para contribuir a la diversificación de contenidos audiovisuales al alcance de los jóvenes antofagastinos (chilenos), de 18 a 29 años, con y sin educación superior, haciendo énfasis en lo cultural-educativo, al desarrollo audiovisual, en el contexto de fortalecer la docencia e investigación en Periodismo de varias Universidades chilenas (Católica de la Santísima Concepción, Católica de Valparaíso, Católica del Norte). En este contexto, la primera transmisión experimental de ULTUR TV (Soñar TV) fue realizada el 21 de enero 2013 (www.youtube.com/watch?v $=\mathrm{hv} 3 \mathrm{GA} 4 \mathrm{vaA} 3 \mathrm{~s}$ ).

Este trabajo pretende, por tanto, indagar en cómo diseñar una IPTV universitaria que incorpore las múltiples posibilidades interactivas y comunicativas de Internet con contenidos audiovisuales innovadores, producidos especialmente para la web en el ámbito de lo científico y divulgativo, con la realización de reportajes y programas televisivos en periodismo científico y divulgación científica (Bueno, 1985: 1.4201.427),(Torrales, 1999: 35-54). «En este proceso de socialización de la ciencia y la tecnología, los medios de comunicación juegan un papel que va mucho más allá de la 
mera difusión de contenidos científicos: contribuyen a crear una imagen pública de la ciencia, de los científicos y de sus implicaciones sociales» (Alcíbar, 2004: 43-70). En ese marco, el estudio indagó en el público meta de la IPTV (estudiantes universitarios, jóvenes profesionales, aquellos sin formación en Universidades e Institutos Profesionales, de 18 a 29 años), sus intereses y necesidades de información, que servirán de base para la elaboración de contenidos audiovisuales, que serán transmitidos por la IPTV.

\section{Estado del arte}

La televisión por Internet forma parte de los tres escenarios futuros para la TV en 2015 (Meyer, 2006: 93-108). 1) la TV móvil mediante teléfonos móviles/celulares y otros aparatos multimedia; 2) los portales de televisión interactivos, con programación lineal (transmisión) y no lineal de contenidos audiovisuales; 3) la era del «egocasting», con plataformas personalizadas de TV, servicios de vídeo bajo demanda, con contenidos personalizados, que permitirán a los espectadores contribuir con tópicos de interés. En este ámbito se sitúa la IPTV.

Según Meyer (2006), la programación en línea de la televisión irá perdiendo fuerza y surgirá una TV alternativa universal, debido a los avances tecnológicos, entre éstos, programas audiovisuales especialmente diseñados para su transmisión vía web. El cambio parece inevitable. Hay indicios de que más de $60 \%$ de los hogares europeos están casi seguros de tener una conexión de banda ancha en 2015 (para ver televisión por Internet). En Francia, el 25\% de sus habitantes recepcionaban ya desde hace años programas televisivos en modos diferentes: tanto el receptor tradicional como con Internet o la telefonía móvil (Renou, Dague \& Louvet, 2007). Complementariamente, ya no es posible ver un tajo entre la World Wide Web y la televisión, dado que ambas se superponen para el entretenimiento, el ocio, la información social, el trabajo y la información general (Ferguson \& Perse, 2000: 155-174). Ambos medios mutuamente se complementan, más que excluirse (Benassini, 2009: 78-79), lo que demuestra la plena convergencia entre televisión e Internet. Así, puede hablarse ya del «cibertelenauta», que desarrolla habilidades para la búsqueda en la Red de contenidos televisivos y que posteriormente participa en la Red opinando sobre sus series favoritas mediante foros de discusión y blogs.

La aparición de los vlog (videosblogs) hace posible un nuevo modelo de TV con contenidos hiperespecializados para comunidades interesadas en tópicos específicos. Más aún, es probable que estemos caminando hacia un nuevo paradigma de la televisión (Meyer, 2006: 93-108). El sector de TV en los próximos años podría tender hacia un modelo alternativo de crecimiento, caracterizado por: a) El boom de programas de TV ofertados por los espectadores de televisión en Internet, b) La mejora de vídeos online y de los servicios de TV desarrollados por los principales grupos de medios, c) El reconocimiento del $\mathrm{P} 2 \mathrm{P}$ y del podcasting como serios canales alternativos para distribuir TV sobre Internet.

E1 P2P (peer-to-peer) es una red informática entre iguales, que permite intercambiar y compartir archivos audiovisuales entre usuarios activos, de una forma más eficiente. Considera una arquitectura descentralizada, donde una serie de nodos se 
comportan simultáneamente como clientes y como servidores respecto de los demás nodos de la red. Este sistema fue popularizado por Napster, Kazaa y otros (Roux, 2006: 48-50).

El podcasting, recurso ya utilizado en la educación (Campbell, 2005: 32-46), consiste en la creación de archivos de sonido, generalmente en formato mp3 y de vídeo, denominados videocasts o vodcasts, que se distribuyen mediante un sistema de sindicación que permite suscribirse y usar un programa que lo descarga de Internet para que el usuario lo escuche y/o vea en el momento que quiera, generalmente en un reproductor portátil. Un avance en este sentido se ha producido con la iniciativa de Apple, que enmarcada en iTunes, nos hace posible derribar las fronteras que limitan el aprendizaje en el terreno del vídeo digital (Aguaded \& Ponce-Guardiola, 2012). Con la aparición de iTunes-U, que se presenta en mayo de 2007, nace una «plataforma de referencia para publicar contenido educativo gratuito en formato digital. Se trata de un medio revolucionario de difusión por Internet mediante el cual instituciones educativas y universidades pueden ofertar contenidos en audio y vídeo, permitiendo realizar búsquedas, descargas, reproducción y sindicación de los materiales de formación de un modo fácil». La importancia e interés de iTunes U se muestra con la integración de 669 universidades, entre las que se encuentran 26 españolas, 21 francesas, 2 brasileñas o 14 mexicanas, y que están aportando contenidos multimedia, documentación escrita, vídeos, audio y materiales de apoyo en los últimos seis años.

El cambio hacia este nuevo modelo de concebir la televisión es ya una realidad, tanto en los espectadores como en los propios canales de producción. En España están en desarrollo proyectos de IPTV, en Madrid (Universidad Complutense): «Complumedia» (http://complumedia.ucm.es) y «e-televisión» (http://e-television.es/canales), la Universidad Nacional de Educación a Distancia (Canal UNED) (http://uned.agilecontents.com/\#frontaleID=F_RC\&sectionID $=S$ _TELUNE\&videoID=7609); y otras experiencias como las de la Asociación de Internautas (www.internautas.tv). En Barcelona funciona una IPTV en el distrito de Gracia (www.graciamon.cat). Todas estas iniciativas están generando repositorios de productos audiovisuales y la creación de canales temáticos: para niños (www.teleclip.tv), recursos de apoyo a la educación universitaria... En suma, una nueva mirada crítica sobre la sociedad de la información y un acceso a Internet accesible y de calidad, utilizando la convergencia de la Red y la televisión.

En Francia opera «TV Demain» (www.demain.fr), la televisión del mañana, iniciativa que forma parte de una cadena tradicional de televisión. Dedicada al empleo y la empresa, tiene cobertura en toda Europa, y transmite iniciativas en emprendimiento, búsquedas de empleo, informaciones para sus espectadores, así como tests que permiten interactivamente a los usuarios conocer mejor sus capacidades para la incorporación al mercado laboral.

En Chile se encuentra en marcha el Canal de Televisión del Consorcio de Universidades del Estado de Chile (www.uestev.cl) que transmite su programación vía Internet. En la iniciativa participan la Red Universitaria Nacional (REUNA) y 16 universidades (Tarapacá, Arturo Prat, Antofagasta, Atacama, La Serena, Valparaíso, Playa Ancha), además de Santiago de Chile, Tecnológica Metropolitana, Metropoli- 
tana, Talca, Bío-Bío, La Frontera, Los Lagos y Magallanes). Esta plataforma consiste en un sistema de digitalización, compresión, almacenamiento y distribución de programas de televisión.

Este estudio analiza el diseño de una IPTV atractiva visualmente, dirigida y realizada por jóvenes, con un tratamiento renovador de los contenidos, producidos especialmente para la web e incorporando las ventajas de Internet y de la IPTV.

\section{La IPTV y sus características distintivas}

La IPTV, como sistema de distribución por suscripción de señales de televisión y/o vídeo, mediante conexiones de banda ancha sobre el protocolo IP (comunica datos en una red de paquetes conmutados), fue desarrollada basándose en el video-streaming (posibilita ver u oír los archivos directamente en una página web sin necesidad de descarga previa). Se considera que tendrá un gran impacto y para ello son necesarias unas redes mucho más rápidas que las actuales, para poder garantizar la calidad en el servicio.

Este medio, a diferencia de la WebTV, que opera con el sistema de transmisión emisor-receptor (broadcasting), incorpora la modalidad P2P, propia de la Web 2.0. Su modelo de distribución contempla una red de dispositivos que cumplen al mismo tiempo las funciones de cliente y servidor (Gálvez, 2007). En ella los participantes no sólo reciben contenidos, sino que también pueden aportarlos, constituyéndose así en usuarios activos de esta modalidad de televisión por Internet. Esta característica tiene una consecuencia de carácter social, indica Valdés (2009) basándose en Castells (2001), al señalar que la sociabilidad basada en el lugar es desplazada por otra que surge debido a las afinidades e intereses comunes, que influencian el intercambio y el consumo de esos bienes simbólicos.

Sin embargo, paralelamente a la situación indicada, con la IPTV y la convergencia tecnológica, «la IPTV adquiere una importante nivel de personalización, que contribuye a que los integrantes del grupo familiar puedan disfrutar de contenidos diferentes de manera independiente» (Gálvez, 2007). Además, superando las complejidades técnicas del ancho de banda, puede llegar a tener más abonados y con mayor resolución de imagen, superior a la difundida por la WebTV.

\section{Jóvenes y consumo de nuevos medios}

La relación de este grupo etario de los jóvenes con las tecnologías de información y comunicación (TIC) es bien compleja (Aguaded, 2013). Si bien «existe la idea que para los jóvenes esta alta penetración de las nuevas tecnologías constituye casi una condición natural de la vida» (Contreras, 2007), para conocer el cómo piensan y qué preferencias tienen, es necesario comprender de forma rigurosa sus ambientes de vida y sus complejas y variadas relaciones con las TIC. García y Rosado (2012) estudiaron los intereses de estos nativos digitales (jóvenes nacidos en un entorno socio-digital que utilizan las redes como un espacio natural, social y personal de su actividad) a diferencia de los inmigrantes digitales (jóvenes ajenos a esta realidad del Internet social). En Francia, «la mayor parte de los jóvenes se sienten poco interesados en los temas que se abordan, ellos rechazan los comentarios y las tomas de posición dema- 
siado marcadas. Además tienen el sentimiento de que los medios les proporcionan lecciones, les imponen una manera de pensar. Ellos van directamente a buscar la información y privilegian los hechos» (Lipani, en Varios, 2011).

Así por ejemplo, en el contexto español, Sabés (2005) señala que, en Aragón, los jóvenes de 16 a 26 años consumen habitual y mayoritariamente televisión $(92 \%$ de hombres y $93 \%$ de mujeres), hecho repetido en todos los niveles de estudios y en todas las situaciones laborales. Quienes pasan más tiempo frente al receptor son los jóvenes sin empleo, «un $55 \%$ de los cuales lo hace dos horas o más, y que los que estudian y trabajan tienen cierta tendencia a concentrar básicamente esta actividad de lunes a viernes». Los jóvenes ven televisión, principalmente para ocupar el tiempo libre, en más del $80 \%$ de los casos, en tanto cerca del $12 \%$ de ellos lo hace como primera prioridad para mejorar su formación.

Para los jóvenes, la televisión «podrá pasar de ser un medio que se recibe pasivamente y que atiende a las decisiones de programación de los programadores tradicionales, a ser un medio en el que los propios usuarios: contribuyan a la creación, seleccionen las fuentes, extraigan y agreguen sus propios contenidos audiovisuales, editen segmentos de programación y promuevan fórmulas de interacción e intercambios múltiples» (García-Matilla \& Molina, 2008: 15-38).

En cuanto a Internet, un $86 \%$ de hombres y un $75 \%$ de mujeres, según Sabes (2005) en esta muestra aragonesa se conectan con cierta regularidad. Su motivación, en comparación con la radio y la televisión, es utilizar la Red como una importante herramienta para la formación y los estudios $(37 \%$ de los hombres pone una de estas opciones en primer lugar). Sin embargo, la mayor parte de los jóvenes (43\% de varones y $55 \%$ de las mujeres) estima que la Red es un entretenimiento para el tiempo libre. Igualmente, la Fundación Española para la Ciencia y Tecnología (FECYT) en su encuesta de percepción social de la Ciencia detectó que el interés de los españoles ha ido creciendo un $36 \%$ en 2010.

En Chile, el consumo de televisión también es muy elevado: un $72 \%$ de los jóvenes entre 18 y 29 años ve TV todos los días, en tanto que un 54\% escucha radio y solamente un 21\% lee la prensa escrita (Encuesta ICSO-UDP, 2007). El uso diario de Internet entre los jóvenes entre 15 y 29 años es de $42 \%$. Complementariamente, el $15,8 \%$ de quienes tienen entre 18 y 24 años utilizan la Red habitualmente para leer noticias y el $24,8 \%$ la usan con cierta frecuencia para ese fin. Este consumo aumenta a un 53,3\% cuando se trata de acceder a información cultural y de espectáculos (Arriagada, Schuster \& Martín, 2008: 34-41).

En ese contexto, y teniendo en cuenta el público meta de la IPTV universitaria, la investigación buscó detectar los contenidos preferenciales de los jóvenes en los programas televisivos de divulgación científica, así como sus percepciones ante las potencialidades de este nuevo medio interactivo como es la IPTV para la comunicación pública de la ciencia y tecnología. Sin duda, la ciencia es una parte importante de la cultura y en ella desempeña un papel fundamental la divulgación científica, que tiene el compromiso de hacer circular socialmente el saber, estimulando con ello la curiosidad y el asombro, fomentando la capacidad crítica y el debate sobre los asuntos controvertidos con implicancias sociales (Alcíbar, 2004: 43-70). 


\section{Material y métodos}

Se utilizó el método cuantitativo. El diseño de investigación fue no experimental transeccional descriptivo. El instrumento de recolección de datos fue un cuestionario que contempló preguntas cerradas y la escala Likert, abordando los ítems como consumo actual de televisión e Internet, el futuro de la TV, los programas de divulgación científica, y el diseño de una IPTV dirigida a acercar la ciencia al público en general. La encuesta tuvo como base los utilizados por Meyer (2006) y Ferguson y Perse (2000), además de ítems originales sobre contenidos preferidos en IPTV y formatos del nuevo medio.

La muestra fue probabilística estratificada y la integraron jóvenes chilenos de la región de Antofagasta de 18 a 29 años, distribuidos en tres segmentos, estudiantes universitarios, jóvenes profesionales, sin formación superior. Su tamaño consistió en 185 sujetos para el primer tramo (46,5\%), 100 personas para el segundo $(25,3 \%)$ y 113 individuos para el tercero (28,3\%), sumando 398 jóvenes. La población en el rango etario citado, según datos del Instituto Nacional de Estadísticas (Censo 2002) fue de 63.970 personas. La tabulación se efectuó con el software SPSS, versión 19.

En cuanto al procedimiento, la aplicación del cuestionario se realizó en octubre y noviembre 2010, controlado por los investigadores y administrado por cinco colaboradores, previamente formados exprofesso.

\section{Análisis y resultados}

Un porcentaje igual o superior a $75 \%$ de los jóvenes antofagastinos, en los tres estratos, consideran que la IPTV predominará en el futuro de la televisión digital. Así, al sumar las opciones muy de acuerdo (MA) y de acuerdo (DA), el 83,1\% sin estudios superiores expresan esta opinión, (MA: 35\%; DA: 51,8\%); en tanto también lo hacen el $82,1 \%$ de los estudiantes universitarios (MA: 33,3\%; DA: 48,9\%) y el 75\% de los jóvenes profesionales (MA: 18\%; DA: 57\%).

En cuanto al estudio, también hubo respuesta positiva de los jóvenes en los tres segmentos. Al ser consultados si una IPTV de divulgación científica responderá a las necesidades de los usuarios, el 90,2\% de los alumnos y alumnas de educación superior, $79 \%$ de los jóvenes profesionales y el $84,8 \%$ de aquellos sin formación superior, estimaron estar muy de acuerdo y de acuerdo con esa afirmación (figura 1).

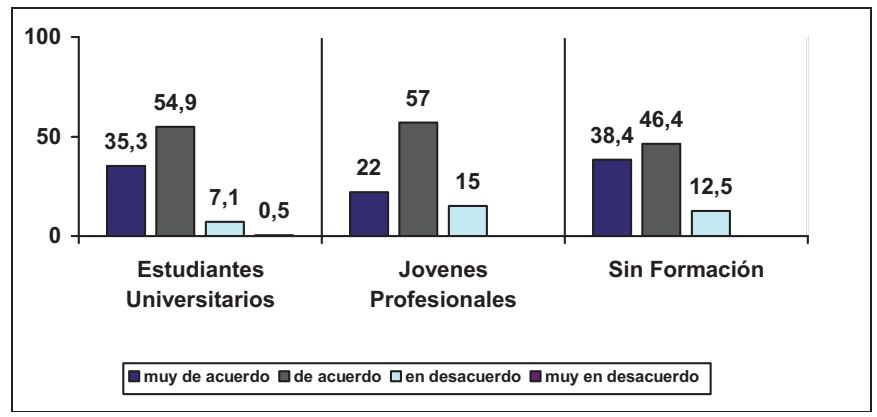

Figura 1: Una IPTV de divulgación científica logrará satisfacer las necesidades de información de los usuarios 
Una situación similar se registró al preguntarles si una IPTV de divulgación científica ayudará al acercamiento de la ciencia a la sociedad, que es justamente uno de los propósitos del diseño del proyecto.

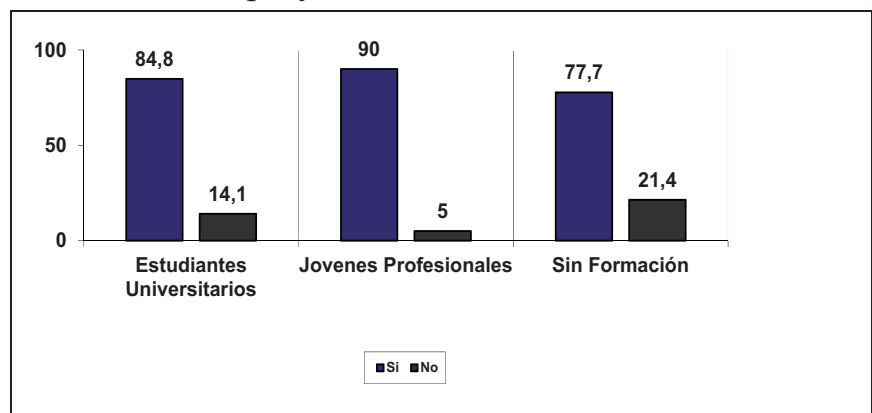

Figura 2: Una IPTV de divulgación científica ayudará al acercamiento entre la ciencia y la sociedad

En materia de consumo de los medios, el 22,3\% de los jóvenes sin formación superior ve televisión solo por Internet y el $4,5 \%$ ve TV e Internet al mismo tiempo. Esa situación difiere levemente con los estudiantes universitarios y de otros centros de formación superior, quienes en un 31\% ven televisión solamente por la Red y el 6,1\% ven Internet y TV en forma simultánea. Los jóvenes profesionales ven televisión solamente por Internet en un 26\% y TV e Internet al mismo tiempo en un $2 \%$.

\begin{tabular}{|c|c|c|c|c|}
\hline \multicolumn{5}{|c|}{$\begin{array}{l}\text { Tabla 1. Contenidos preferidos un programa } \\
\text { de IPTV de divulgación científica }\end{array}$} \\
\hline Estrato & \multicolumn{2}{|c|}{ wwwContenidos } & Frecuencia & Porcentaje \\
\hline \multirow{10}{*}{$\begin{array}{l}\text { Sin formación } \\
\text { superior }\end{array}$} & Válidos & Salud y medicina & 43 & 38,4 \\
\hline & & Educación & 37 & 33,0 \\
\hline & & Medioambiente & 4 & 3,6 \\
\hline & & Tecnología & 17 & 15,2 \\
\hline & & Astronomía & 4 & 3,6 \\
\hline & & Sismología & 2 & 1,8 \\
\hline & & Otro & 4 & 3,6 \\
\hline & & Total & 111 & 99,1 \\
\hline & Perdidos & Sistema & 1 & ,9 \\
\hline & \multicolumn{2}{|l|}{ Total } & 112 & 100,0 \\
\hline \multirow{10}{*}{$\begin{array}{l}\text { Estudiante } \\
\text { universitario }\end{array}$} & Válidos & Salud y medicina & 61 & 33,2 \\
\hline & & Educación & 58 & 31,5 \\
\hline & & Medioambiente & 21 & 11,4 \\
\hline & & Tecnología & 27 & 14,7 \\
\hline & & Astronomía & 6 & 3,3 \\
\hline & & Sismología & 6 & 3,3 \\
\hline & & Otro & 4 & 2,2 \\
\hline & & Total & 183 & 99,5 \\
\hline & Perdidos & Sistema & 1 &, 5 \\
\hline & \multicolumn{2}{|l|}{ Total } & 184 & 100,0 \\
\hline
\end{tabular}




\begin{tabular}{|c|c|c|c|c|}
\hline \multirow{9}{*}{ Joven Profesional } & Válidos & Salud y medicina & 32 & 32,0 \\
\hline & & Educación & 36 & 36,0 \\
\hline & & Medioambiente & 7 & 7,0 \\
\hline & & Tecnología & 11 & 11,0 \\
\hline & & Astronomía & 2 & 2,0 \\
\hline & & Sismología & 1 & 1,0 \\
\hline & & Otro & 6 & 6,0 \\
\hline & & Total & 95 & 95,0 \\
\hline & Perdidos & Sistema & 5 & 5,0 \\
\hline & \multicolumn{2}{|l|}{ Total } & 100 & 100,0 \\
\hline
\end{tabular}

La encuesta permitió además detectar los contenidos que a los jóvenes antofagastinos les gustaría ver en un programa de divulgación científica. Se constató que les interesan contenidos de salud y medicina $(38,4 \%, 33,2 \%$ y $32 \%$, en los estratos sin formación superior, estudiantes universitarios y jóvenes profesionales, respectivamente), la educación (33\%, 31,5\% y 36\% en los mismos segmentos), la tecnología $(15,2 \%, 14,7 \%$ y $11 \%$, correspondientemente) y el medio ambiente concentra también la preferencia del público meta $(3,6 \%, 11,4 \%$ y $7 \%$, en el mismo orden). Ver Tabla 1.

Dado el interés de generar en la IPTV contenidos de divulgación científica en ámbitos como la actualidad, los documentales, la ficción, se consultó a los jóvenes sobre el tipo de programación que les gustaría ver en el nuevo medio. Hay algunas diferencias entre los tres segmentos. En los estudiantes universitarios, por ejemplo, las preferencias son para los contenidos musicales $(28,26 \%)$, entretenimiento $(26,63 \%)$, documentales de divulgación científica $(25 \%)$, documentales socioculturales $(19,56 \%)$, reportajes de divulgación científica $(19 \%)$, cortometrajes $(18,47 \%)$, deportes $(16,84 \%)$ y programas de divulgación científica $(12,5 \%)$.

\begin{tabular}{|l|r|r|r|r|r|r|}
\hline \multirow{2}{*}{\begin{tabular}{c}
\multirow{4}{*}{$\begin{array}{c}\text { Categorías y } \\
\text { formatos }\end{array}$} \\
\cline { 2 - 8 }
\end{tabular}} & \multicolumn{2}{c|}{$\begin{array}{c}\text { Estudiantes } \\
\text { universitarios }\end{array}$} & \multicolumn{2}{c|}{$\begin{array}{c}\text { Jóvenes } \\
\text { Profesionales }\end{array}$} & \multicolumn{2}{c|}{$\begin{array}{c}\text { Sin formación } \\
\text { profesional }\end{array}$} \\
\cline { 2 - 8 } (N:184) & \multicolumn{2}{c|}{$\begin{array}{c}\text { (N:100) } \\
\text { (N:112) }\end{array}$} \\
\hline $\begin{array}{l}\text { Reportajes de } \\
\text { divulgación científica }\end{array}$ & 35 & 19,0 & 24 & 24 & 15 & 13,39 \\
\hline $\begin{array}{l}\text { Documentales } \\
\text { científicos }\end{array}$ & 46 & 25,00 & 8 & 8 & 17 & 15,17 \\
\hline $\begin{array}{l}\text { Programas de } \\
\text { divulgación científica }\end{array}$ & 23 & 12,50 & 26 & 26 & 9 & 8,00 \\
\hline Cortometrajes & 34 & 18,47 & 27 & 27 & 2 & 1,78 \\
\hline $\begin{array}{l}\text { Documentales } \\
\text { socioculturales }\end{array}$ & 36 & 19,56 & 12 & 12 & 20 & 17,85 \\
\hline Contenidos musicales & 52 & 28,26 & 12 & 12 & 28 & 25,00 \\
\hline Ficción & 16 & 8,69 & 4 & 4 & 15 & 13,39 \\
\hline Sociedad & 16 & 8,69 & 9 & 9 & 8 & 7,14 \\
\hline Entretenimiento & 49 & 26,63 & 20 & 20 & 30 & 26,78 \\
\hline
\end{tabular}




\begin{tabular}{|l|r|r|r|r|r|r|}
\hline Noticieros & 11 & 5,97 & 6 & 6 & 6 & 5,35 \\
\hline Deportes & 31 & 16,84 & 4 & 4 & 11 & 9,82 \\
\hline Política & 7 & 3,80 & 1 & 1 & 3 & 2,67 \\
\hline Economía & 8 & 4,34 & 2 & 2 & 1 & 0,89 \\
\hline Cultura & 18 & 9,78 & 5 & 5 & 6 & 5,35 \\
\hline Videojuegos & 9 & 4,89 & & & 1 & 0,89 \\
\hline
\end{tabular}

\section{Discusión y conclusiones}

Los nuevos medios pueden ser una magnífica oportunidad de aproximar la ciencia a la sociedad. La explosión del conocimiento, gracias a la investigación científica y tecnológica, puede generar brechas importantes (Aguaded, 2013) si no se acompasa con la divulgación del saber. Es para ello necesario que la comunidad se apropie de los nuevos saberes y procedimientos para utilizarlos en su vida cotidiana, aumentando su participación cívica fundamentada y el aumento de su cultura científica, en línea con los propósitos socializadores del periodismo científico (Alcíbar, 2004: 43-70). Especialmente significativo será alcanzar estas metas en las nuevas generaciones, los jóvenes.

El trabajo de campo permitió verificar que la decisión de crear e implementar una IPTV universitaria es una decisión apoyada en las necesidades de los jóvenes, ya que éstos coinciden en señalar que ese medio prevalecerá en el futuro de la televisión digital, respaldando así uno de los tres escenarios propuestos por Meyer. Ello nos permite inferir que los establecimientos de educación superior deben apostar en incorporar este nuevo medio de comunicación en los procesos internos de comunicación, así como en los procesos de enseñanza-aprendizaje de los alumnos, especialmente cuando éstos están basados en modelos de formación por competencias.

Una modalidad de TV por Internet para jóvenes, con producción televisiva realizada por jóvenes generará al mismo tiempo las ventajas de acceso y uso responsable de Internet y las múltiples posibilidades de interacción comunicativa que puede generar una IPTV en el ámbito de la divulgación científica, con tratamientos innovadores, que serían atractivos para el público meta, por la cercanía que tienen con las tecnologías de información y comunicación y sus distintas motivaciones (Sabés, 2005), (Arriagada, Schuster \& Martín, 2008: 34-41). Con esta generación de contenidos se responderá de forma focalizada a los intereses y necesidades informativas de este sector etario (18 a 29 años), a veces tan ignorado por los medios. La IPTV universitaria giraría en este campo de acción.

Salud y medicina, educación, tecnología, medio ambiente son los contenidos que los jóvenes chilenos de Antofagasta, de 18 a 29 años, les gustaría ver preferentemente en programas de divulgación científica. En los estratos, sin formación superior y estudiantes universitarios destaca la opción por salud y medicina ( $43 \%$ y $61 \%$, respectivamente), siendo esto coherente con el entorno y el período que viven. La ciudad históricamente ha tenido dificultades en salud, vinculadas con medio ambiente (contaminación por plomo, comestibles regados con aguas no apropiadas, arsénico en el agua para consumo humano, entre otros). La educación alcanza también porcentajes significativos, especialmente en los alumnos universitarios (58\%), quienes dan igual- 
mente prioridad a la tecnología (27\%), presentes en su vida cotidiana y en los establecimientos de enseñanza superior. Un estudio posterior podría detectar los temas urgentes a divulgar en cada uno de esos ámbitos.

En términos de generación de contenidos es significativo tener identificadas las distintas categorías y formatos preferidos por jóvenes de los tres estratos. Los estudiantes universitarios y sin formación superior enfatizan los documentales de divulgación científica ( $25 \%$ y $15,17 \%$, respectivamente) y los de tipo sociocultural $(19,56 \%$ y $17,85 \%$, en ese orden). Los cortometrajes son elegidos luego por alumnos y alumnas de enseñanza superior y jóvenes profesionales $(18,47 \%$ y $27 \%$, correspondientemente), quienes prefieren también los programas de divulgación científica (12,5\% y $26 \%$, en ese orden). Cabe resaltar los altos porcentajes que alcanzan entretenimiento, superior al $26 \%$ en los tres estratos y contenidos musicales, igual o superior a $25 \%$ en los tres niveles de formación.

Los resultados del estudio nos permiten, sin duda, redireccionar de mejor forma la gestión y realización de proyectos audiovisuales, contribuyendo al enriquecimiento cultural y educativo del público joven; conforman también un impacto en la docencia universitaria, factible de incrementar con otras investigaciones académicas, que favorezcan la formación por competencias de los alumnos de la Universidad.

\section{Referencias bibliográficas}

AGUADED, José Ignacio (2013): "Media Programme (UE) - International Support for Media Education". Comunicar, 40, pp. 7-8. (DOI: 10.3916/C40-2013-01-01).

AGUADED, José Ignacio \& PONCE-GUARDIOLA, Daniel (2012): “UniTV, canal de televisión de la Universidad de Huelva". Edmetic, 1, 1, pp. 94-117.

ALCÍBAR, Miguel (2004): "La divulgación mediática de la ciencia y la tecnología como recontextualización discursiva". Análisi, 31, pp. 43-70. En: http://ddd.uab.cat/pub/analisi/02112175n31p43.pdf) [Consultado: 15-04-2013]

ARRIAGADA, Arturo \& SCHUSTER, Martin (2008): "Consumo de medios y la participación ciudadana de los jóvenes chilenos". Cuadernos de Información, 22, pp. 34-41.

BENASSINI, Claudia (2009): Televisión por Internet. México (DF), Alfaomega.

BUENO, Wilson (1985): "Jornalismo científico. Conceito e funçoes". Ciencia e Cultura, 37 (9), pp. 1420-1427.

CAMPBELL, Gardner (2005): There's Something in the Air. Podcasting in the Education. Educase Review, pp. 32-46. En: www.cblt.soton.ac.uk/multimedia/PDFs08 /Podcasting\%20in\%20education.pdf) [Consultado:16-03-2013]

CASTELLS, Manuel (2001): La Galaxia Internet. Reflexiones sobre Internet, empresa y sociedad. Barcelona, Plaza \& Janes Editores.

CONTRERAS, David (2007): "Las y los jóvenes en las TIC: formas de uso que hablan de nuevas culturas y nuevas brechas". Observatorio de la Juventud, nuevos perfiles generacionales. 4 (15), 50. Madrid, Instituto Nacional de la Juventud. 
CORREDOIRA, Loreto (1998): "Consecuencias de la convergencia. Las «push technologies», un nuevo modo de difusión”. En CORREDOIRA, Loreto (Ed.): Los retos de la información en Internet. Las libertades de acceso y difusión. Madrid, Seminario Complutense de Telecomunicaciones e Información.

FAGUNDES, Antonio (2006): "Uso do video on-line como síntoma de alternativa para a TV na era digital". XXIX Congreso Brasileiro de Ciencias de Comunicacao, 6 a 9 de setembro Universidade Nacional de Brasilia (Brasil), Intercom, Sociedade Brasileira de Estudios Interdisciplinares da Comunicacao.

FERGUSON, Douglas \& PERSE, Elizabeth (2000): "The World Wide Web as a Functional Alternative to Television". Journal of Broadcasting \& Electronic Media, 44 (2), pp. 155-174.

GÁLVEZ, Javier (2007): IPTV. Televisión digital interactiva sobre IP. Barcelona, Telecom.

GARCÍA, Francisco y ROSADO, María Jesús (2012): "Conductas socio-comunicativas de los nativos digitales y los jóvenes en la web 2.0". Comunicación y Sociedad, 25 (1), pp. 15-38.

GARCÍA-MATILLA, Agustín y MOLINA, Juan Pedro (2008): “Televisión y jóvenes en España”. Comunicar, 31, pp. 83-90. (DOI:10.3916/c31-2008-01-0010).

LA VANGUARDIA.COM (2010): "Aumenta el consumo cultural de los jóvenes gracias a las nuevas tecnologías": www.lavanguardia.com/ciudadanos/noticias/20100910/54000966163/aumenta-el-consumo-cultural-de-los-jovenes-gracias -a-las-nuevas-tecnologias.html) [Consultado: 01-09-2011]

MAMANI, Pablo; MENESES, Cristian; MONTECINOS, Jesús; RAMOS, Rodrigo; y RODRÍGUEZ, Alejandro (2010): IPTV y programas de divulgación científica: explorando nuevos formatos. Tesis de pre-grado no publicada. Antofagasta (Chile), Escuela de Periodismo, Universidad Católica del Norte.

MEYER, Laurence (2006): “Three Scenarios for TV in 2015". Communications \& Strategies, 62, pp. 93-108: http://ssm.com/abstract=992853[ Consultado: 25-092011]

RENOU, Aymeric; DAGUE, Thierry; \& LOUVET, Bertrand Régis (2007): "Internet change notre façon de regarder la télé". Le Parisen (Paris), 23 fevrier, 2; édition de Paris.

ROUX, Dominique (2006): TV et video sur Internet. Paris, Económica, pp. 48-50.

ROUX, Dominique (2007): La Televisión de demain. Paris, Económica.

SABÉS, Fernando (2005): "La televisión que quieren los jóvenes: hacia un medio de proximidad". Comunicar, 25: www.revistacomunicar.com/verpdf.php?numero $=25 \&$ articulo $=25-2005-067$ [Consultado:16-04-2013].

TOBÓN, Sergio (2004): Formación basada en competencias. Pensamiento complejo, diseño curricular y didáctica. Bogotá, ECOE. 
TORRALES, Daniel (1999): Periodismo científico y nueva educación. Antofagasta, Ediciones Universitarias UCN, pp. 35-54.

TORRALES, Daniel y MATUS, Mauricio (2009): “Televisión 2.0. WebTV e IPTV: nuevos recursos para la formación universitaria en gestión y producción en televisión, vídeo y cine". Tercer Milenio, 14 (17), 54-61: www.tercermilenio.ucn.cl /e17/tercermilenio17/art05.html [Consultado:18-07-2011].

VALDÉS, Claudio (2009): “Televisión 2.0: consumo y producción audiovisual”. En CARVAJAL, Julio y RODRÍGUEZ, Gustavo (Eds.): Comunicación informativa y nuevas tecnologías. Buenos Aires, Gran Aldea Editores, pp. 43-61. 